\title{
Autopsy of the Supernova Remnant Cassiopeia A
}

\author{
Dan Milisavljevic ${ }^{1}$ and Robert A. Fesen ${ }^{2}$ \\ ${ }^{1}$ Harvard-Smithsonian Center for Astrophysics, 60 Garden Street, Cambridge, MA, 02138 \\ email: dmilisav@cfa.harvard.edu \\ ${ }^{2} 6127$ Wilder Lab, Dept. of Physics \& Astronomy, Dartmouth College, Hanover, NH 03755 \\ email: robert.fesen@dartmouth.edu
}

\begin{abstract}
Three-dimensional kinematic reconstructions of optically emitting ejecta in the young Galactic supernova remnant Cassiopeia A (Cas A) are discussed. The reconstructions encompass the remnant's faint outlying ejecta knots, including the exceptionally high-velocity NE and SW streams of debris often referred to as 'jets'. The bulk of Cas A's ejecta are arranged in several circular rings with diameters between approximately $30^{\prime \prime}(0.5 \mathrm{pc})$ and $2^{\prime}(2 \mathrm{pc})$. We suggest that similar large-scale ejecta rings may be a common phenomenon of young core-collapse remnants and may explain lumpy emission line profile substructure sometimes observed in spectra of extragalactic core-collapse supernovae years after explosion. A likely origin for these large ejecta rings is post-explosion input of energy from plumes of radioactive ${ }^{56} \mathrm{Ni}$-rich ejecta that rise, expand, and compress non-radioactive material to form bubble-like structures.
\end{abstract}

Keywords. supernova remnant

\section{Introduction}

Massive stars $\left(\geqslant 8 \mathrm{M}_{\odot}\right)$ explode as core-collapse supernovae (SNe; Types II, IIb, Ib,c) and are a primary source of heavy elements in the universe. The specific engine that drives these explosions is uncertain, but gravity along with aspherical influences from uneven neutrino heating, rotation, and magnetic fields have been investigated (see Janka 2012 and references therein). Debate over the dynamics and relative strength of these influences stems in large part from a dearth of observational constraints.

Study of the class of young oxygen-rich supernova remnants (SNRs) believed to be the end products of SNe provide opportunities to directly test models of massive stellar explosions. Nearby SNRs permit investigations of explosion kinematics and elemental abundances at spatial scales that are impossible to achieve from extragalactic SN observations, and thus yield unique information about the core collapse explosion mechanism, nucleosynthesis yields, and the nature of their central compact remnants.

The young Galactic remnant Cassiopeia A (Cas A) provides perhaps the clearest look at the explosion dynamics of a high mass SN. With an explosion date most likely around $1681 \pm 19$, Cas A is the youngest Galactic core-collapse SNR known (Thorstensen et al. 2001; Fesen et al. 2006), and at an estimated distance of $3.4 \mathrm{kpc}$ (Reed et al. 1995), it is also among the closest.

Cas A is the only historical core-collapse SNR with a secure SN subtype classification. The detection of light echoes of the supernova outburst (Rest et al. 2011; Besel et al. 2012) has enabled follow-up observations which indicate that the original supernova associated with Cas A exhibited an optical spectrum at maximum light similar to those seen for the Type IIb events SN 1993J and SN 2003bg (Krause et al. 2008; Rest et al. 2011). 


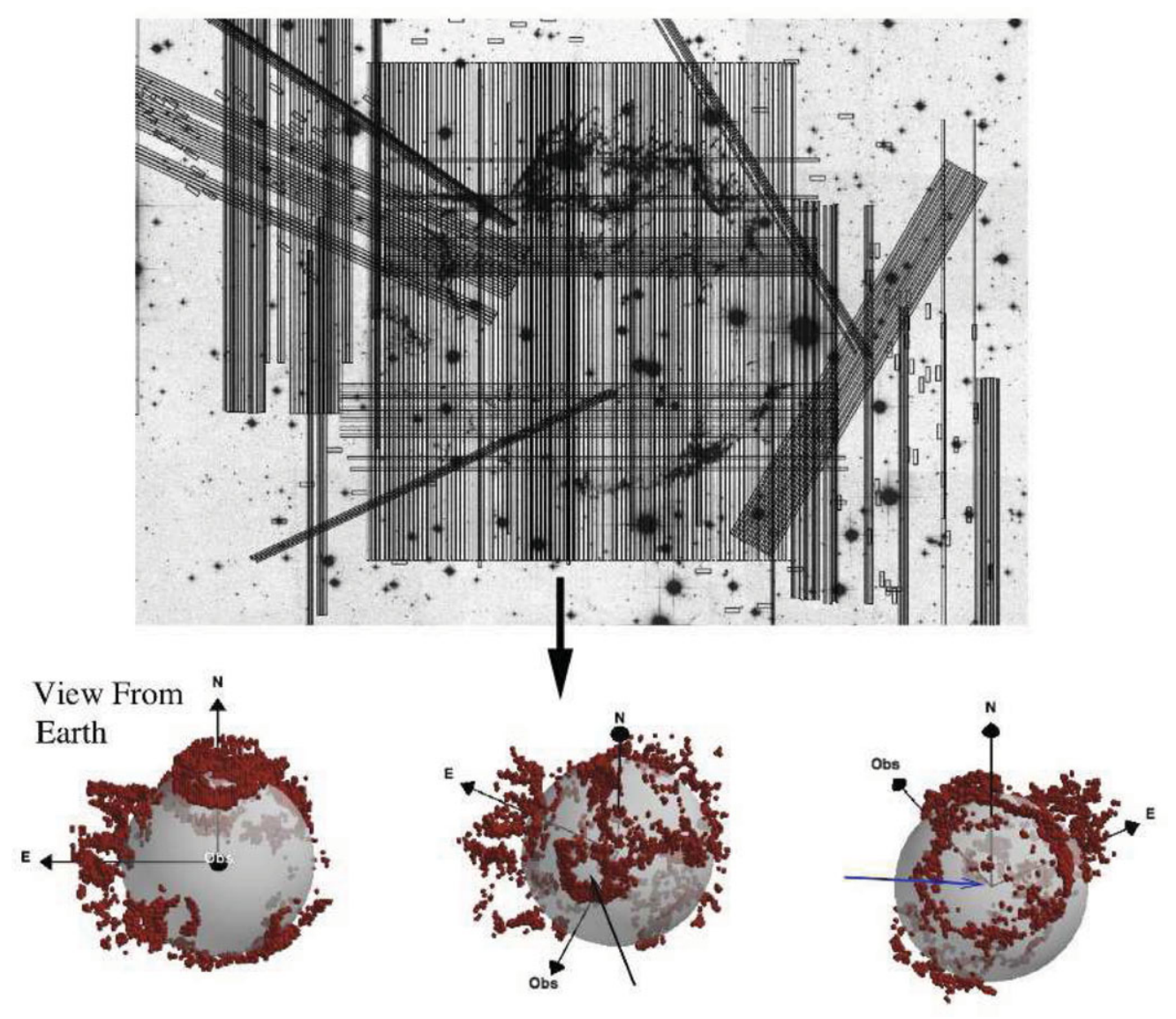

Figure 1. Data from Milisavljevic \& Fesen (2013) showing the kinematic map constructed for Cas A. Top: Locations of over 200 slit positions used to develop a 3D reconstruction. Bottom: Various perspectives of the resulting 3D map. Blue arrow points to a region coincident with X-ray emitting Fe-rich material.

\section{3D Reconstruction of Cas A}

We recently created a detailed 3D kinematic reconstruction of Cas A. Its opticalemitting ejecta were mapped from a spectroscopic survey involving hundreds of long slit spectra taken over three years (Milisavljevic \& Fesen 2013; Figure 1). It is the most complete kinematic map of an SNR to date.

Our data show that Cas A's main shell ejecta are arranged in several well-defined and nearly circular rings with diameters between approximately $30^{\prime \prime}(0.5 \mathrm{pc})$ and $2^{\prime}(2 \mathrm{pc})$. In Figure 2, a Mercator projection of the main shell knots is shown to illustrate the relative scale and distribution of the rings. Some rings form complete circles, while others appear as partial circles or ellipses. Three of these rings encircle X-ray-emitting Fe-rich ejecta that has been mapped by DeLaney et al. (2010).

Motivation to undertake a deep reconnaissance of the entire Cas A was driven in part from an interest in finally grasping the kinematic properties of the NE and SW jets. Previous studies of Cas A's NE and SW regions of exceptionally high-velocity knots could not address the question of whether they form a true bipolar structure. Our data indicate that this is indeed the case. The NE and SW jets with expansion velocities approaching $15,000 \mathrm{~km} \mathrm{~s}^{-1}$ appear to be directed in nearly opposite directions suggestive of opposing 


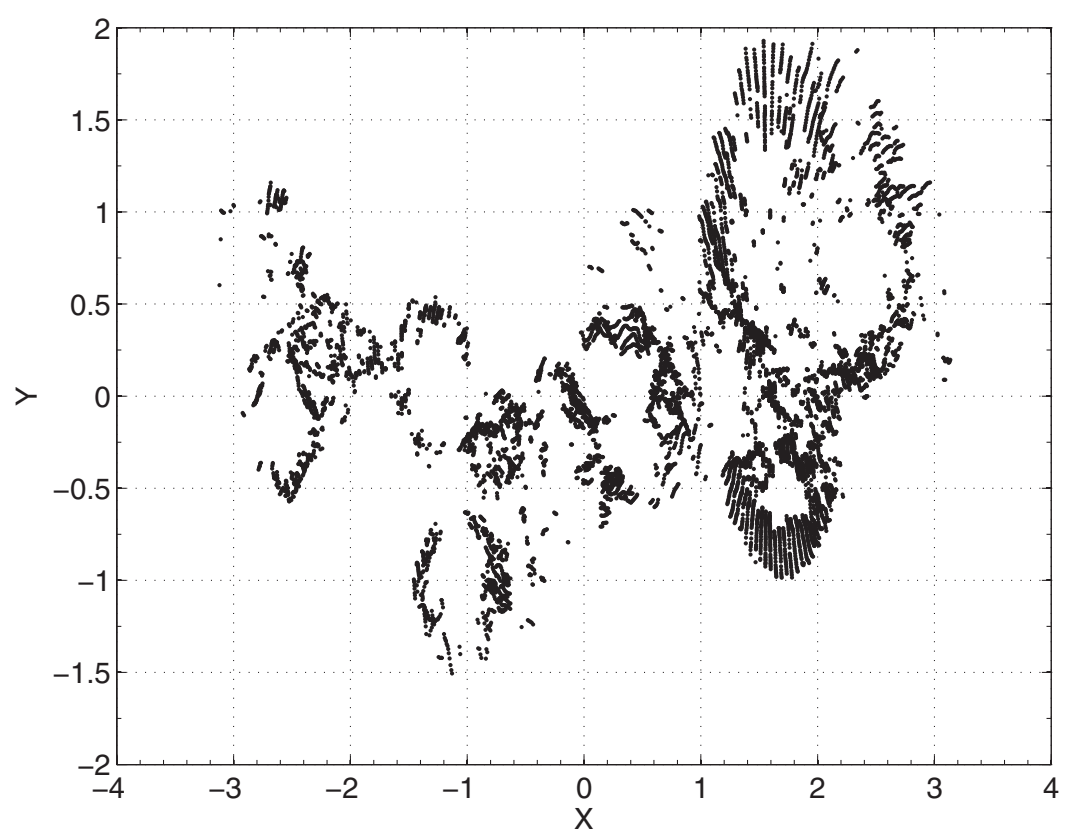

Figure 2. The main shell of Cas A's optically-emitting ejecta as represented in a Mercator projection. The linear scale is equal in all directions around any point and conformal, but the cylindrical map projection distorts the size and shape of large objects, especially towards the poles. From Milisavljevic \& Fesen (2013).

flows of SN debris originating from the Si-S-Ca-Ar layer of the progenitor star with opening half-angles of approximately 40 degrees.

\section{Discussion}

It is clear from both our survey and previous studies that the distribution of Cas A's ejecta is not random. At least half a dozen large and coherent ejecta rings are observed. The sizes and arrangement of these rings may be informing us about important properties of the explosion dynamics and subsequent evolution of the expanding debris. We note, however, that interpretation of these structures is complicated in that one must disentangle properties that may originate in the explosion from later influences related to possible post-explosion radioactive heating, ejecta interaction with the surrounding material, and effects of the reverse shock on the ejecta.

The ejecta rings are not unlike large-scale features seen in SN explosion models (e.g., Hammer et al. 2010) and may have origin in part to a "Ni bubble effect" having influenced the remnant's expansion dynamics shortly after the original explosion. It may be that the observed ejecta rings represent cross-sections of large cavities in the expanding ejecta created by a post-explosion input of energy from plumes of radioactive ${ }^{56} \mathrm{Ni}$-rich ejecta. Li et al. (1993) have described how this input of energy might account for the high-volume filling factor of Fe in SN 1987A despite its small mass, and Basko et al. (1994) and Blondin et al. (2001) have investigated hydrodynamic simulations based on this model. In this scenario the compression of surrounding non-radioactive material by rising and expanding bubbles of radioactive ${ }^{56} \mathrm{Ni}$-rich ejecta occupy a large fraction of the ejecta volume and give way to a "Swiss cheese" ejecta structure. 
We find that the overall conical distributions of the NE and SW jet regions exhibit comparable maximum expansion velocities and are broadly anti-parallel in an orientation consistent with a bipolar jet-counterjet structure. However, the observed opening halfangles of these two flows (approximately 40 degrees) is quite broad and is not what would be anticipated in a highly-collimated (opening half-angle $<10$ degrees) jet-induced explosion (e.g., Khokhlov et al. 1999; Wheeler et al. 2002; Akiyama et al. 2003). Future work mapping the chemical abundances of the jet knots (several thousand in number) to their kinematics might contribute to a better understanding of their nature and possible relationship to the main ejecta shell.

\section{Broader Implications}

Our results with Cas A may have implications for other young core-collapse remnants. Milisavljevic et al. (2012) summed all our main shell spectra into a single, integrated spectrum. This mimicked what the remnant would appear as as an unresolved extragalactic source. Intriguingly, close similarities were found between the integrated Cas A spectrum and several late-time optical spectra of decades-old extragalactic SNe, including SN 1979C, SN 1993J, SN 1980K, and the ultra-luminous supernova remnant in NGC 4449.

These spectra all showed pronounced blueshifted emission with conspicuous line substructure in [O I] $\lambda \lambda 6300,6364,[\mathrm{O}$ II] $\lambda \lambda 7319,7330,[\mathrm{O}$ III] $\lambda \lambda 4959,5007,[\mathrm{~S}$ II] $\lambda \lambda 6716$, 6731 , and [Ar III] $\lambda 7136$. Since the emission line substructure observed in the forbidden oxygen emission line profiles of Cas A are associated with the large-scale rings of ejecta, we suggest that similar features in the intermediate-aged SNe which have often been interpreted as ejecta 'clumps' or 'blobs' are, in fact, probable signs that similar large-scale rings of ejecta are common in SNe.

\section{Acknowledgements}

Some of this material is based upon work supported by the National Science Foundation under Grant No. AST-0908237.

\section{References}

Akiyama, S., Wheeler, J. C., Meier, D. L., \& Lichtenstadt, I. 2003, ApJ, 584, 954

Basko, M. 1994, ApJ, 425, 264

Besel, M.-A. \& Krause, O. 2012, A\&A, 541, L3

Blondin, J. M., Borkowski, K. J., \& Reynolds, S. P. 2001, ApJ, 557, 782

DeLaney, T., Rudnick, L., Stage, M. D., et al. 2010, ApJ, 725, 2038

Fesen, R. A., Hammell, M. C., Morse, J., et al. 2006, ApJ, 645, 283

Hammer, N. J., Janka, H., \& Müller, E. 2010, ApJ, 714, 1371

Janka, H.-T. 2012, Annual Review of Nuclear and Particle Science, 62, 407

Khokhlov, A. M., Höflich, P. A., Oran, E. S., et al. 1999, ApЛ, 524, L107

Krause, O., Birkmann, S. M., Usuda, T., et al. 2008, Science, 320, 1195

Li, H., McCray, R., \& Sunyaev, R. A. 1993, ApJ, 419, 824

Milisavljevic, D. \& Fesen, R. A. 2013, ApJ, submitted

Milisavljevic, D., Fesen, R. A., Chevalier, R. A., et al. 2012, ApJ, 751, 25

Reed, J. E., Hester, J. J., Fabian, A. C., \& Winkler, P. F. 1995, ApJ, 440, 706

Rest, A., Foley, R. J., Sinnott, B., et al. 2011, ApJ, 732, 3

Thorstensen, J. R., Fesen, R. A., \& van den Bergh, S. 2001, AJ, 122, 297

Wang, L., Wheeler, J. C., Höflich, P., et al. 2002, ApJ, 579, 671

Wheeler, J. C., Meier, D. L., \& Wilson, J. R. 2002, ApJ, 568, 807 


\section{Discussion}

Unidentified: 1. Are the "rings" in the projection plot dominated by 1 element, or do they all have same/very similar composition? 2. Are the bigger rings moving faster? Could they have been ejected BEFORE the explosion from the progenitor?

MilisavlJEviC: The map shown in purely kinematics and does not show any information about chemical abundances. The material is generally all at the same radius from the center of expansion so everything being seen now should have originated at the same time of explosion.

TuRATto: Is there any alignment or connection between the so-called jets and planes in $\mathrm{X}$-rays that you have shown?

MiLisAVLJEVIC: Interesting question. There is no clear relationship.

MAEDA: Have you got an idea to get the 'integrated' O profile from the unshocked ejecta? You did it for the 'shocked' ejecta, but it might be affected by ISM/CSM ?

MilisavlJevic: This is a good idea! One could use the "bubble-like" interior as a map to simulate a clumpy distribution of radioactive elements that could power emission from say, oxygen and calcium. One could then compare this to the late-time emission line profiles observed in supernovae 1 year after outburst.

BARTEL: What is the proper motion of the X-ray point source inferred from the difference between its position and the extrapolated position of the center of expansion. Further, you mentioned that the proper motion is almost perpendicular to the jet direction. What physical significance would that have?

MilisavlueviC: The inferred proper motion is something like $350 \mathrm{~km} / \mathrm{s}$. I have not yet come up with an explanation of why this should be so. In fact, it run counter to what once would expect if the NE \& SW flares were associated with a jet-induced explosion if this were the case the motion of the neutron star should be along the jet axis. 